\title{
Screening for the Prevention of Cervical Cancer in the Era of Human Papillomavirus Vaccination: An Australian Perspective
}

\author{
Annabelle Farnsworth \\ Douglass Hanly Moir Pathology, Sydney, N.S.W., Australia
}

\author{
Key Words \\ Cervical screening $\cdot$ Pap smears $\cdot$ Human papillomavirus \\ vaccination · Sensitivity $\cdot$ Positive predictive value $\cdot$ Pap \\ test registers
}

\begin{abstract}
Australia has a unique and highly successful screening program for cervical cancer which is based on the conventional Pap smear. Since its introduction in 1991 there has been a decline in both the incidence of and mortality from this disease. Part of the success of this program has been the introduction of Pap test registers and strict quality assurance measures for cervical cytology, including compulsory key performance indicators for laboratories. Using these measures, nationwide calculations give cervical cytology in Australia a sensitivity of $78 \%$ for high-grade lesions and a positive predictive value (PPV) of $78 \%$. Australia was the first country to introduce a widespread government-funded human papillomavirus (HPV) vaccination program in 2007. Because of the high accuracy of Australian cytology, HPV testing alone, given its low PPV and high cost, is unlikely to be a viable alternative to cytology for primary screening in this country. Australia therefore faces unique issues and choices in integrating its extensive vaccination program with a successful cervical screening program.
\end{abstract}

Copyright $\odot 2011$ S. Karger AG, Basel

\begin{tabular}{ll}
\hline KARGER & (c) 2011 S. Karger AG, Basel \\
Fax +41613061234 & $0001-5547 / 11 / 0554-0307 \$ 38.00 / 0$ \\
$\begin{array}{l}\text { E-Mail karger@karger.ch } \\
\text { www.karger.com }\end{array}$ & $\begin{array}{l}\text { Accessible online at: } \\
\text { www.karger.com/acy }\end{array}$
\end{tabular}

\section{Introduction}

With the introduction of vaccination against two of the oncogenic strains of human papillomavirus (HPV), government-funded population-based screening programs for the prevention of cervical cancer need to be reevaluated.

Programs for cervical cancer screening, although widespread, particularly in western developed countries, differ in many of their elements. Screening intervals, screening populations and screening tests vary from country to country and even within countries. There are also differences in recruitment strategies, funding, recording of relevant epidemiological data and research approaches. Most countries have also taken individual approaches to HPV vaccination programs. Choice of vaccine, selection of target groups and the funding of the vaccination program vary among countries. In some, vaccination programs are either fully or partially funded by the government, whilst in others HPV vaccines have still not been licensed.

When decisions are being made regarding the integration of vaccination and screening, each country will need to take a different approach depending on the nature of their preexisting programs. Most importantly, any changes to screening programs need to be appropriately assessed taking outcomes and cost-effectiveness into consideration.

Australia has a unique successful screening program which has been in place for 20 years. The incidence of

Correspondence to: Dr. Annabelle Farnsworth

Douglass Hanly Moir Pathology

14 Giffnock Ave., Macquarie Park 2113

Sydney, NSW 2113 (Australia)

Tel. +61 29855 5180, E-Mail afarnsw@dhm.com.au 


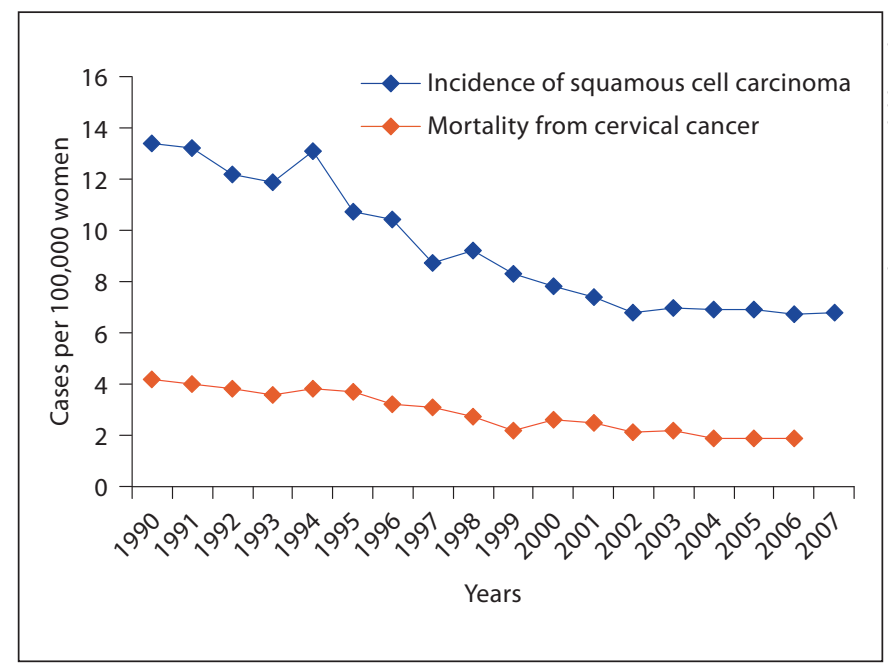

Fig. 1. Incidence of squamous cell carcinoma of cervix in Australia from 1990 to 2007 (age-standardized) versus trend in mortality from cervical cancer from 1990 to 2006.

cervical cancer has decreased substantially with the current rate at 6.9/100,000 women and the mortality at $1.4 / 100,000$ [1] (fig. 1). It was also the first country to introduce HPV vaccination in 2007. Although there have already been a number of reviews of the impact of HPV vaccination on cervical screening [2-4], this paper addresses some of the issues relevant in a country with a well-established and highly successful screening program as well as an extensive vaccination regime.

\section{The Current Australian Screening Program}

An organized approach to screening for the prevention of cervical cancer was introduced in Australia in 1991. The recommended screening interval is 2-yearly, commencing at age 18 and ceasing at age 70 . The screening test is the conventional Pap smear, although it is estimated that approximately $20-30 \%$ of all conventional smears are accompanied by a liquid-based cytology sample, still prepared as a split-sample test. There is a government rebate for the conventional smear but not for the separate liquid-based cytology sample. Unlike in the USA [5], HPV testing is not recommended for primary screening. A government subsidy applies only to HPV 'test-ofcure' after treatment for high-grade lesions. HPV testing is also performed where clinically indicated or at the request of a patient, but no government rebate is available for such tests.

\section{Pap Test Registers}

One of the unique aspects of the Australian cervical screening program was the introduction of Pap test registers (PTR). These record all cervical cytology, histology and, more recently, HPV test results. They are opt-off registers so, unless the patient actively declines, it is compulsory for all laboratories to forward test results to these central registers. The opt-off rate is less than $1 \%$ and for these women only deidentified results are recorded. The registers are not 'call-and-recall' registers but do offer safety net follow-up programs. They send out reminder letters to both clinicians and women, provide statistics for monitoring the program and also provide laboratories with information which enables them to complete compulsory quality audits.

\section{Quality Assurance Measures}

All laboratories reporting cervical cytology as part of the National Cervical Screening Program must be assessed by the Royal College of Pathologists of Australia (RCPA)/National Australian Testing Authority (NATA) joint program in order to have access to government funding.

To be accredited, laboratories are subject to regular NATA inspections and must meet a set of laboratory requirements which are set by the National Pathology Accreditation \& Advisory Council (NPAAC) [6]. There are many requirements ranging from scientific qualifications, ongoing education and limits on the maximum number of slides to be screened by individuals. Laboratories must also be enrolled in an external quality assurance program.

In addition, laboratories reporting cervical cytology must meet specific performance indicators. These are a set of key, quantifiable criteria that allow comparisons of performance of individual laboratories. The data are collected by laboratories with the help of PTR, and reported annually to the RCPA Quality Assurance Program. The performance standards were last revised in 2005. A review of a decade (1998-2008) of data derived from these performance measures was recently published [7].

As well as being a key factor in the development of high standards in cervical cytology, summary data from these performance measures provide comparisons of the effectiveness of cervical cytology in Australia over time. Two of the four measures in particular (Performance Measures 3 and 4) can be used as nationwide estimates of the accuracy of cervical cytology.

Performance Measure 3. This measure reports the percentage of cases where histology taken within 6 months 
of a cytologic report of a high-grade squamous intraepithelial abnormality (HSIL) or adenocarcinoma in situ (AIS) confirms the abnormality as an HSIL, AIS or cancer. This therefore provides the positive predictive value (PPV) of cervical cytology. In 2009 the value, Australiawide, was $78 \%$ [7].

Performance Measure 4. This measure reports data on the accuracy of previous negative smear results from women with histologically proven high-grade abnormalities. It provides an estimate of the sensitivity of cervical cytology. Australia-wide data showed that $22 \%$ of women with histological diagnosis of high-grade abnormality in 2009 had had a negative cytology result in the 30 months preceding the histology. This sensitivity of $78 \%$ for cervical cytology is significantly higher than the oft-quoted $50-60 \%$ [7]. On review of the slides, the proportion of women with negative smears, who were identified as screening false negatives, has averaged $14 \%$ over the last 6 years. When comparing the various estimates of sensitivity in the literature, one has to bear in mind that different methods, populations and assumptions were used in their calculations. The Australian estimate quoted here is population-based, clearly defined and comprehensive.

\section{The Australian Vaccination Program}

Australia was the first country to introduce a universal vaccination program using the quadrivalent vaccine against two of the oncogenic HPV subtypes, 16 and 18 . The vaccination program was introduced in 2007 to both a cohort of schoolgirls in their first year of high school (aged 12-13 years) as well as to a catch-up group of women up to the age of 26. This vaccination program was fully funded by the government. The school program is ongoing, but the catch-up program ceased on 31 December 2009.

Because of the potential benefits of this vaccine, its introduction proceeded even though there were, and still are, a number of unanswered questions: How long would immunity last? Would boosters be necessary? What screening regime would be appropriate for the vaccinated cohort of women and how would this be integrated into the current screening program? To help answer some of these issues, a vaccination register was also introduced to ensure records were kept of vaccination status and to record rates of immunizations throughout the country [8]. Vaccination uptake records have not yet been released but are thought to be high in the schoolgirl cohort group and lower in the catch-up group.

Cervical Screening in the Era of HPV Vaccination
As the vaccine targets HPV 16 and 18, it is of interest to know the incidence of these subtypes in Australia. Recently released data show the rate of HPV 16 in the general population to be $8.3 \%$, with a small number being HPV 18 positive [9]. Another study shows the rates of HPV 16 and 18 in a population of women presenting to a colposcopy clinic with abnormal Pap smears to be 35 and $8 \%$, respectively [10].

The immediate effect of the vaccination program on the reduction in cervical disease is still unknown and may remain so for many years. Modelling studies predict a significant fall in the incidence of HPV 16 [11]. The fully vaccinated population is mostly still very young and comprises only a small percentage of the screening population. The expected fall in high-grade lesions is not yet evident. Our laboratory has certainly seen no recent fall in the rates of reporting high-grade cervical cytology. Although there is as yet no evidence of the reduction in cervical disease, recent data [12] show that since the introduction of the quadrivalent vaccine, there has been a reduction in the incidence of genital warts at an Australian sexual health clinic. This quadrivalent vaccine, in addition to HPV types 16 and 18, contains types 6 and 11 which are quite specific for this type of genital infection.

For a number of reasons vaccination alone will be insufficient to completely protect women from the development of cervical cancer. There will be mixed immunity amongst Australian women for some years, due to both the variation in vaccination uptakes, and the large group of women over the age of 27 who will continue to need screening for many years. Further, HPV 16 and 18 are not the only high-risk types, not all vaccinated women build up immunity, some women refuse vaccination and immigrant women are unlikely to have been vaccinated. In fact, vaccination alone would offer less protection than the current screening program [13]. A combination of screening and vaccination will therefore continue to be necessary.

\section{Screening for Cervical Cancer in the Era of HPV Vaccination}

When decisions are being made about cervical cancer screening in a vaccinated population, the major issues to consider are the screening test, the age of commencement of screening and the screening interval. Another significant decision is whether to offer different screening programs to different populations depending on their vaccination and immune status, using data from the HPV Vaccine Register.

Acta Cytologica 2011;55:307-312 309 
It may be too confusing and difficult to send mixed messages to the screening population. Recruitment in Australia is already a significant issue. The biennial screening rates in some of the major jurisdictions are as low as $56 \%$ [14]. If, in future, the screening program is no longer universal, due consideration will need to be given to appropriate education strategies.

\section{Which Screening Test?}

The best test for screening for cervical cancer continues to be debated internationally for both the vaccinated and unvaccinated populations. The debate centers mostly on the value of using HPV testing versus cervical cytology.

\section{HPV Testing as a Primary Screening Tool}

There has now been a number of large published trials comparing the efficacy of HPV testing with cervical cytology in primary screening for the prevention of cervical cancer [15-17]. Most of the HPV test studies have occurred either in situations with an unknown quality of cytology or, alternatively, low standards of cervical cytology. The sensitivity for cervical cytology is usually quoted at $50-60 \%[18,19]$. The significantly higher sensitivity of HPV testing, upwards of $90 \%$, therefore appears advantageous when compared with cervical cytology.

Tests suitable for population-based screening have different requirements to those used for clinical testing. It became apparent over the years that strict parameters and quality indicators had to be applied to cervical cytology to be effective as a screening test. In Australia, the introduction of performance measures, together with the establishment of PTR, has resulted in a successful screening program in terms of outcomes and cost-effectiveness. Any new screening test will need to be subjected to the same quality measures as cervical cytology.

\section{Estimate of Sensitivity of HPV Tests in New \\ South Wales}

Data from the New South Wales (NSW) PTR were used to estimate the sensitivity of HPV testing using the same criteria as Performance Measure 4 for cervical cytology.

PTR in Australia have been collecting HPV test data prospectively since 2008 and retrospectively from June 2005. Cross-sectional data were extracted from the NSW PTR for women who had histological high-grade CIN $2-3$, or AIS or invasive cancers reported in the period of
Table 1. Percentage of negative HPV tests and the interval by which the HPV tests precede high-grade histology

\begin{tabular}{lcc}
\hline & HPV tests, $\mathrm{n}$ & HPV negative \\
\hline Concurrent & 709 & $11 \%$ \\
$<6$ months & 745 & $9 \%$ \\
$6-12$ months & 203 & $14 \%$ \\
$12-24$ months & 265 & $22 \%$ \\
$24-30$ months & 80 & $29 \%$ \\
\hline All & 2,002 & $13 \%$ \\
\hline
\end{tabular}

1 January 2008 to 30 September 2009, and also had had an HPV test, either concurrently with or in the 30 months preceding that high-grade histology. The information was extracted as deidentified data, and ethics approval was given by the NSW Cancer Institute.

In all, 2,002 HPV tests were retrieved from 1,683 women. The results are summarized in table 1, classified according to the time between the HPV test and the subsequent histology. Overall, 13\% of tests were HPV negative and $15 \%$ of women had had at least one negative HPV test. Of note is that $29 \%$ of the tests taken in the period $24-30$ months prior to the known high-grade histology were reported as negative for HPV.

The results have also compared HPV tests preceding different types of cervical abnormality. Thirteen percent of HSIL had negative HPV tests, whereas, although numbers are small, $37 \%$ of AIS and $80 \%$ of endocervical adenocarcinomas had negative HPV tests preceding the histological diagnosis (table 2).

In summary, overall, $15 \%$ of women had a negative HPV test (vs. 22\% with a negative cytology) within 30 months preceding high-grade lesions. The rate of negative HPV tests increased significantly with time between HPV test and histology, and at 24-30 months from the biopsy, the rate was $29 \%$. A higher percentage of glandular lesions had preceded negative HPV tests. We do not have a similar breakdown of previous cervical cytology for statewide NSW for comparison. However, in 2008/2009 there were 4,485 women with histological high-grade reports who had cytology reported in our own laboratory in the preceding 30 months. A breakdown of these results is included in table 2. These figures show that, although the sensitivity of cytology is also lower for glandular than for squamous lesions, cytology was more sensitive than HPV for glandular lesions. Even for histological HSIL, the difference in the relative sensitivities of cytology and HPV tests (78 vs. $87 \%$ ) is not as great as one would have expected. 
Table 2. Percentage of negative HPV and cytology tests within 30 months preceding the histology

\begin{tabular}{lrlr}
\hline $\begin{array}{l}\text { Subtype of } \\
\text { high-grade histology }\end{array}$ & $\begin{array}{l}\text { HPV } \\
\mathrm{n}\end{array}$ & $\begin{array}{l}\text { HPV } \\
\text { negative }\end{array}$ & $\begin{array}{l}\text { Cytology } \\
\text { negative }\end{array}$ \\
\hline HSIL & 1,920 & $13 \%$ & $22 \%$ \\
SCC & 26 & $8 \%$ & $14 \%$ \\
HSIL + AIS & 32 & $13 \%$ & $9 \%$ \\
AIS & 19 & $37 \%$ & $28 \%$ \\
Endocervical adenocarcinoma & 5 & $80 \%$ & $40 \%$ \\
\hline
\end{tabular}

HPV tests based on NSW PTR data; cytology tests based on DHM laboratory data. SCC $=$ Squamous cell carcinoma.

It should be noted that the type of HPV testing used in these reports was not known, although Digene Hybrid Capture is the most commonly used HPV test in NSW.

The reason for having the HPV tests was also unknown, but few would have been for primary screening as the government rebate is only for 'test-of-cure'. The implication of this is difficult to determine - are HPV false negatives more or less likely to occur in women with previous abnormalities than in screening populations?

These results, although only from one state in Australia, certainly encourage further investigation to be undertaken comparing high-quality cervical cytology with HPV testing. The final choice will of course also need to take the relative costs of the tests into consideration. Currently, in Australia, the cost of HPV testing is about 3 times that of cytology.

False Positives: Specificity and PPV

The accuracy of a screening test, however, is not solely dependent on its sensitivity. The value of a screening test will always be a balance between false negatives and false positives. The latter must be considered, as false positives will result in unnecessary investigations and waste of scarce health dollars. Sensitivity is a well-accepted measure of the false-negative rate. Specificity is often used as the measure of the false-positive rate. However, when the prevalence of disease is low, as in the case for cervical disease, comparisons of specificity can be misleading. The calculation of specificity has such a large denominator, i.e. the whole screening population, that very small differences in specificity can mask large differences in the actual number of false positives [2]. The PPV is a superior indicator of the relative false-positive rates in such situations.
The most commonly used HPV test, Digene Hybrid Capture, has a low PPV [20]. Although this may still be a valid clinical test as a screening test, this low PPV can create significant problems. Further, in a situation of low prevalence, such as in a vaccinated population, the PPV will be even lower [21].

\section{Cervical Cytology as a Primary Screening Tool}

The low sensitivity of cervical cytology worldwide is commonly reported [19]. There has also been criticism that cytology will be even less accurate with lower sensitivity and PPV in a situation of even lower prevalence such as would be seen in a vaccinated population [22].

Another method of screening in a vaccinated population is automated cervical cytology. Any system that allows prescreening of the sample when prevalence is low would present selected material to the cytologist for visual inspection and analysis. The value of liquid-based cytology continues to be debated [23]. However, one large study from Australia showed increased sensitivity using ThinPrep with Imager [24].

Any comparison of screening tests should also include evaluations of new technologies for cervical cytology.

\section{New Technologies for Cervical Screening}

It is apparent that the current tests for HPV which probe for a mixture of high-risk subtypes will alone not fulfill screening criteria in either the vaccinated or nonvaccinated population.

Different regimes, including delayed onset of screening, using cytology triage and lengthening the screening interval have been suggested to address the issue of low PPV and cost. New tests offering specific HPV subtyping are now available and may be of benefit particularly in the vaccinated population.

A new generation of tests is emerging which look not just for the presence or absence of HPV but for specific markers of neoplastic cell activity. Many of these have been reviewed elsewhere [25] and numerous technologies are still being developed. Most recently, a technique for analyzing samples using flow cytometry has been published [26].

However, it is beyond the scope of this review to look at the large number of tests which are being developed and proposed as possible alternative screening tests for cervical cancer. From the Australian perspective, any of these new tests will need to be properly assessed and compared with our current successful screening strategy in terms of both accuracy and cost-effectiveness. 


\section{Conclusion}

Australia has both a highly successful cervical screening program and an extensive government-funded HPV vaccination program. Integration of these two entities will be needed to achieve the best outcomes for Australian women in the most cost-effective manner.

The high standards of Australian cytology will make simplistic adoption of any 'new' screening test difficult. The commonly used high-risk HPV cocktail test is of value in the way in which it is currently used, as either a testof-cure or a triage of equivocal cytology in older women.
However, because of its low PPV and relatively high cost, this test is unlikely to be suitable as a screening test at present.

\section{Acknowledgment}

I would like to thank the NSW PTR for their cooperation in the provision of the HPV data.

\section{Disclosure Statement}

The author has no conflict of interest to declare.

\section{References}

1 Australasian Association of Cancer Registries, Australian Government: Australian Institute of Health and Welfare, Canberra, 2010. http://www.aihw.gov.au/cancer/data/ acim books/cervical_cancer.xls.

2 Schiffman M: Integration of human papillomavirus vaccination, cytology, and human papillomavirus testing. Cancer Cytopathol 2007;111:145-153.

-3 Heideman D, Snijders PJ, Berkhof J, Verheijen RH, Helmerhorst TJ, Meijer CJ: Vaccination against $\mathrm{HPV}$ : indications for women and the impact on the cervical screening programme. BJOG 2008;115:938-946.

-4 Goldhaber-Fiebert JD, Stout NK, Salomon JA, Kuntz KM, Goldie SJ: Cost-effectiveness of cervical cancer screening with human papillomavirus DNA testing and HPV-16, 18 vaccinations. J Natl Cancer Inst 2008;100: 308-320.

5 Solomon D, Papillo JL, Davey DD: Statement on HPV DNA test utilization. Acta Cytol 2009;53:247-248.

6 National Pathology Accreditation Advisory Council: Requirements for Gynaecological (Cervical) Cytology. Canberra, ACT, 2006.

7 Shield PW, Finnimore J, Cummings M, Wright RG: Performance measures for Australian laboratories reporting cervical cytology: a decade of data 1998-2008. Pathology 2010;42:623-628.

8 National HPV Vaccination Program Register 2001, Victorian Cytology Service Inc, East Melbourne. http://www.hpvregister.org.au.

9 Garland S, Brotherton J, Stevens M, Tabrizi S, Condon J, McIntyre P, Smith D: The results of the WHINURS study (Women HPV Indigenous Non-Indigenous Urban Rural Study): a baseline genotype prevalence prior to vaccine rollout (abstract). Australian and New Zealand Vulvovaginal Society, November 2010.

$>10$ Stevens MP, Garland SM, Tan JH, Quinn MA, Petersen RW, Tabrizi SN: HPV genotype prevalence in women with abnormal pap smears in Melbourne, Australia. J Med Virol 2009;81:1283-1291.
11 Smith MA, Canfell K, Brotherton JM, Lew JB, Barnabas RV: The predicted impact of vaccination on human papillomavirus infections in Australia. Int J Cancer 2008; 123: 1854-1863.

-12 Donovan B, Franklin N, Grulich AE, Regan DG, Ali H, Wand H, Fairley CK: Quadrivalent human papillomavirus vaccination and trends in genital warts in Australia: analysis of national sentinel surveillance data. Lancet Infect Dis 2011;11:39-44.

13 Farnsworth A: The changing landscape for cervical screening. Med J Aust 2008;189:6-7.

14 National Cervical Screening Program: Cervical Screening in Australia 2007-2008 Data Report. Canberra, The Australian Institute of Health and Welfare and the Australian Government Department of Health and Ageing, 2010.

15 Mayrand MH, Duarte-Franco E, Rodrigues I, Walter SD, Hanley J, Ferenczy A, Ratnam S, Coutlee F, Franco EL; Canadian Cervical Cancer Screening Trial Group: Human papillomavirus DNA versus Papanicolaou screening tests for cervical cancer. N Engl J Med 2007;357:1579-1588.

16 Dillner J, Rebolj M, Birembaut P, Petry KU, Szarewski A, Munk C, de Sanjose S, Naucler P, Lloveras B, Kjaer S, Cuzick J, van Ballegooijen $\mathrm{M}$, Clavel C, Iftner T: Long term predictive values of cytology and human papillomavirus testing in cervical cancer screening: joint European cohort study. BMJ 2008; 377:a1754.

17 Sankaranarayanan R, Nene BM, Shastri SS, Jayant K, Muwonge R, Budukh AM, Hingmire S, Malvi, S, Thorat R, Kothari A, Chinoy R, Kelkar R, Kane S, Desai S, Keskar VR, Rajeshwarkar R, Panse N, Dinshaw KA: HPV screening for cervical cancer in rural India. N Engl J Med 2009;360:1385-1394.

18 Wright TC Jr: Cervical cancer screening in the 21st century: is it time to retire the PAP smear? Clin Obstet Gynecol 2007;50:313-323.
19 Nanda K, McCrory DC, Myers ER, Bastian LA, Hasselblad V, Hickey JD, Matchar DB: Accuracy of the Papanicolaou test in screening for and follow-up of cervical cytologic abnormalities: a systematic review. Ann Intern Med 2000;132:810-819.

20 Baleriola C, Millar D, Melki J, Coulston N, Altman P, Rismanto N, Rawlinson W: Comparison of a novel HPV test with the Hybrid Capture II (hcII) and a reference PCR method shows high specificity and positive predictive value for 13 high-risk human papillomavirus infections. J Clin Virol 2008;42: 22-26.

21 Myers E, Huh WK, Wright JD, Smith JS: The current and future role of screening in the era of HPV vaccination. Gynecol Oncol 2008;109:S31-S39.

-22 Franco EL, Cuzick J, Hildesheim A, de Sanjose $S$ : Chapter 20: issues in planning cervical cancer screening in the era of HPV vaccination. Vaccine 2006;24:S3/171-177.

23 Davey E, Barratt A, Irwig L, Chan SF, Macaskill P, Mannes P, Saville AM: Effect of study design and quality on unsatisfactory rates, cytology classifications, and accuracy in liquid-based cytology versus conventional cervical cytology: a systematic review. Lancet 2006;367:122-132.

24 Davey E, d'Assuncao J, Irwig L, Macaskill P, Chan SF, Richards A, Farnsworth A: Accuracy of reading liquid based cytology slides using the ThinPrep Imager compared with conventional cytology: prospective study. BMJ 2007;335:1-8.

25 Dehn D, Torkko KC, Shroyer KR: Human papillomavirus testing and molecular markers of cervical dysplasia and carcinoma. Cancer Cytopathol 2007;111:1-14.

26 Coquillard G, Palao B, Patterson BK: Quantification of intracellular HPV E6/E7 mRNA expression increases the specificity and positive predictive value of cervical cancer screening compared to HPV DNA. Gynecol Oncol 2011;120:89-93. 